\title{
Extension problem for principles of equivalent utility
}

\author{
JACEK CHUDZIAK
}

Dedicated to Professor Karol Baron on the occasion of his 70-th birthday.

\begin{abstract}
We prove that, under the Cumulative Prospect Theory, every principle of equivalent utility, defined on a family of ternary risks, possesses a unique extension to the family of all risks.
\end{abstract}

Mathematics Subject Classification. 39B12, 39B82, 91B16.

Keywords. Principle of equivalent utility, Extension, Insurance premium, General linear equation.

\section{Introduction}

In insurance mathematics, risks are usually represented by non-negative bounded random variables on a given probability space. A premium principle is a functional assigning to every risk a non-negative real number. There are several methods of defining principles. In this paper we deal with the principle of equivalent utility, belonging to the so-called economic methods of insurance contracts pricing. The principle, introduced by Bühlmann [2], involves the notion of a utility function and postulates a fairness in terms of utility. In order to recall the principle, assume that $w \in[0, \infty)$ is an insurance company's initial wealth level and $\preceq$ is its preference relation over a family $\mathcal{X}_{+}$of risks. Then the relation $\preceq$ in a natural way induces on $\mathcal{X}_{+}$the indifference relation $\sim$ :

$$
X \sim Y \Longleftrightarrow(X \preceq Y) \text { and }(Y \preceq X)
$$

for $X, Y \in \mathcal{X}_{+}$. The principle of equivalent utility for the risk $X \in \mathcal{X}_{+}$is a real number $H_{\preceq}(X)$ such that

$$
w+H_{\preceq}(X)-X \sim w .
$$


This condition has the following interpretation: the company is indifferent between rejecting the contract and entering into it. Thus, $H_{\preceq}(X)$ is a minimal price for which the insurance company would be ready to insure the risk $X$.

In general, the principle of equivalent utility need not exist and, even if it exists, it need not be uniquely determined. Under the Expected Utility theory (1) becomes

$$
E\left[u\left(w+H_{u}(X)-X\right)\right]=u(w),
$$

where $u: \mathbb{R} \rightarrow \mathbb{R}$ is a continuous strictly increasing utility function. One can prove that, for every $X \in \mathcal{X}_{+}$, there exists a unique real number $H_{u}(X)$ such that (2) is valid. Therefore, Eq. (2) determines a functional on $\mathcal{X}_{+}$, called the principle of equivalent utility. For more details concerning the properties of the principle of equivalent utility under the Expected Utility Theory we refer e.g. to $[1,2,8,14]$.

The principle of equivalent utility under the Rank-Dependent Utility model and under the Cumulative Prospect Theory, has been introduced and considered by Heilpern [7] and Kałuszka and Krzeszowiec [9,10], respectively. Under the first of these models, the premium $H_{(u, g)}(X)$ for a risk $X \in \mathcal{X}_{+}$is defined as a solution of the equation

$$
E_{g}\left[u\left(w+H_{(u, g)}(X)-X\right)\right]=u(w),
$$

where $u: \mathbb{R} \rightarrow \mathbb{R}$ is a continuous strictly increasing utility function and $E_{g}$ is the Choquet integral with respect to a probability distortion function $g$. Let us recall that $g:[0,1] \rightarrow[0,1]$ is called a probability distortion function, provided it is non-decreasing and satisfies the boundary conditions $g(0)=0$ and $g(1)=1$. For every bounded random variable $X$, the Choquet integral with respect to the probability distortion function $g$ is defined as follows

$$
E_{g}[X]=\int_{-\infty}^{0}(g(P(X>t))-1) d t+\int_{0}^{\infty} g(P(X>t)) d t .
$$

It has been proved in [4] that, if $g$ is a continuous probability distortion function and $u: \mathbb{R} \rightarrow \mathbb{R}$ is a continuous strictly increasing utility function, with $u(0)=$ 0 , then for every $X \in \mathcal{X}_{+}$the number $H_{(u, g)}(X)$ is uniquely determined by (3).

Under the Cumulative Prospect Theory, the premium $H_{(u, g, h)}(X)$ for a risk $X \in \mathcal{X}_{+}$is defined as a solution of the equation

$$
E_{g h}\left[u\left(w+H_{(u, g, h)}(X)-X\right)\right]=u(w)
$$

where, for every bounded random variable $X$,

$$
E_{g h}[X]=E_{g}[\max \{X, 0\}]-E_{h}[\max \{-X, 0\}]
$$

is the generalized Choquet integral related to the probability distortion functions $g$ (for gains) and $h$ (for losses). According to [4, Theorem 3.1], in the case where $w \in(0, \infty)$, for every continuous and strictly increasing function $u: \mathbb{R} \rightarrow \mathbb{R}$ satisfying $u(0)=0$ and every continuous probability distortion 
functions $g$ and $h$, Eq. (5) uniquely determines $H_{(u, g, h)}(X)$ for $X \in \mathcal{X}_{+}$. If $w=0$, then $H_{(u, g, h)}(X)$ is uniquely determined by (5) for every $X \in \mathcal{X}_{+}$if and only if

$$
h(p)+g(1-p)>0 \text { for } p \in[0,1] .
$$

Furthermore (cf. [9]), we have

$$
\begin{gathered}
0 \leq H_{(u, g, h)}(X) \leq \operatorname{ess} \sup X \text { for } X \in \mathcal{X}_{+}, \\
H_{(u, g, h)}\left(c \cdot \mathbb{1}_{\Omega}\right)=c \text { for } c \in \mathbb{R}
\end{gathered}
$$

and

$$
H_{(u, g, h)}\left(X+c \cdot \mathbb{1}_{\Omega}\right)=H_{(u, g, h)}(X)+c \text { for } X \in \mathcal{X}_{+}, c \in[0, \infty) .
$$

These properties are usually referred to as: non-excessive loading, no unjustified risk loading and translation invariance (or consistency), respectively. Furthermore, as the generalized Choquet integral is monotone (cf. [9, Lemma 1]), from (5) one can easily derive that the principle of equivalent utility under the Cumulative Prospect Theory is monotone, that is

$$
H_{(u, g, h)}(X) \leq H_{(u, g, h)}(Y) \text { for } X, Y \in \mathcal{X}_{+}, \quad X \leq Y .
$$

It turns out that, under the Expected Utility model, every functional of equivalent utility can be uniquely extended from the family of all binary risks, i.e. risks taking exactly two non-negative values with positive probabilities, to $\mathcal{X}_{+}$(cf. e.g. [5, Theorem 6]). In fact, in [5] only the case $w=0$ has been considered but, making a straightforward substitution, from [5, Theorem 6$]$ one can easily derive the analogous result for $w>0$. In a recent paper [3], some aspects of the extension problem for functionals of equivalent utility under the Cumulative Prospect Theory have been investigated. In particular, the functionals whose restriction to the family of binary risks reduces either to the net principle or to the exponential principle, have been characterized. It follows from the results in [3] that under the Cumulative Prospect Theory a counterpart of [5, Theorem 6] does not hold. Therefore, the following question naturally arises: does there exist a reasonable, in a sense, family of risks such that, under the Cumulative Prospect Theory, every functional of equivalent utility defined on this family can be uniquely extended to $\mathcal{X}_{+}$. The aim of this paper is to show that the family of ternary risks, taking exactly three non-negative values, one of them being 0 , with positive probabilities, possesses such a property.

\section{Auxiliary results}

Assume that $(\Omega, \Sigma, P)$ is a non-atomic probability space and $\mathcal{X}_{+}$is the family of all non-negative bounded random variables on $(\Omega, \Sigma, P)$. According to $(5)$ the premium for a given risk depends only on a probability distribution of 
the risk. Therefore, in what follows we identify the risks with their probability distributions. Let

$$
\mathcal{P}_{3}:=\left\{\bar{p}=\left(p_{1}, p_{2}, p_{3}\right): p_{1}, p_{2}, p_{3} \in(0,1): p_{1}+p_{2}+p_{3}=1\right\} .
$$

Since $(\Omega, \Sigma, P)$ is non-atomic, for every $x_{1}, x_{2}, x_{3} \in \mathbb{R}$, with $x_{1}<x_{2}<x_{3}$, and every $\bar{p}=\left(p_{1}, p_{2}, p_{3}\right) \in \mathcal{P}_{3}$, there exists a random variable $X$ on the space $(\Omega, \Sigma, P)$ such that $P\left(X=x_{i}\right)=p_{i}$ for $i \in\{1,2,3\}$ (cf. e.g. [12, Lemma 2.7.1]). We denote such a random variable by $\left\langle x_{1}, x_{2}, x_{3} ; \bar{p}\right\rangle$. Furthermore, $\mathcal{X}^{(3)}$ denotes the family of all such random variables and

$$
\mathcal{X}_{0}^{(3)}:=\left\{\langle 0, x, y ; \bar{p}\rangle: 0<x<y, \bar{p} \in \mathcal{P}_{3}\right\} .
$$

In the whole section we assume that $w \in[0, \infty), u: \mathbb{R} \rightarrow \mathbb{R}$ is a strictly increasing continuous function with $u(0)=0$ and $g, h:[0,1] \rightarrow[0,1]$ are continuous distortion functions such that $g(p), h(p) \in(0,1)$ for $p \in(0,1)$. Then (7) is satisfied and so, as we have already noted, the principle of equivalent utility $H_{(u, g, h)}$ is uniquely defined by (5). Applying (4) and (6), we get that, if $X=\left\langle x_{1}, x_{2}, x_{3} ; p_{1}, p_{2}, p_{3}\right\rangle \in \mathcal{X}^{(3)}$, then

$$
\begin{aligned}
& E_{g h}[X]=\left(1-g\left(p_{2}+p_{3}\right)\right) x_{1}+\left(g\left(p_{2}+p_{3}\right)-g\left(x_{3}\right)\right) x_{2}+g\left(p_{3}\right) x_{3} \quad \text { if } \quad x_{1} \geq 0, \\
& E_{g h}[X]=h\left(p_{1}\right) x_{1}+\left(g\left(p_{2}+p_{3}\right)-g\left(p_{3}\right)\right) x_{2}+g\left(p_{3}\right) x_{3} \quad \text { if } \quad x_{1}<0 \leq x_{2}, \\
& E_{g h}[X]=h\left(p_{1}\right) x_{1}+\left(h\left(p_{1}+p_{2}\right)-h\left(p_{1}\right)\right) x_{2}+g\left(p_{3}\right) x_{3} \quad \text { if } \quad x_{2}<0 \leq x_{3}
\end{aligned}
$$

and

$$
E_{g h}[X]=h\left(p_{1}\right) x_{1}+\left(h\left(p_{1}+p_{2}\right)-h\left(p_{1}\right)\right) x_{2}+\left(1-h\left(p_{1}+p_{2}\right)\right) x_{3} \quad \text { if } \quad x_{3}<0 .
$$

Let

$$
T:=\left\{(x, y) \in(0, \infty)^{2}: x<y\right\}
$$

and, for every $\bar{p} \in \mathcal{P}_{3}$, let $\phi_{\bar{p}}, \psi_{\bar{p}}: T \rightarrow \mathbb{R}$ be defined as follows

$$
\begin{aligned}
& \phi_{\bar{p}}(x, y)=w+H_{(u, g, h)}(\langle 0, x, y ; \bar{p}\rangle)-x \quad \text { for } \quad(x, y) \in T, \\
& \psi_{\bar{p}}(x, y)=w+H_{(u, g, h)}(\langle 0, x, y ; \bar{p}\rangle)-y \quad \text { for } \quad(x, y) \in T .
\end{aligned}
$$

Obviously, we have

$$
\psi_{\bar{p}}(x, y)<\phi_{\bar{p}}(x, y) \quad \text { for } \quad(x, y) \in T, \bar{p} \in \mathcal{P}_{3},
$$

and

$$
\psi_{\bar{p}}(x, y)-\phi_{\bar{p}}(x, y)=x-y \quad \text { for } \quad(x, y) \in T, \bar{p} \in \mathcal{P}_{3} .
$$

Moreover, in view of (8), we get

$$
w-x \leq \phi_{\bar{p}}(x, y) \leq w+y-x \quad \text { for } \quad(x, y) \in T, \bar{p} \in \mathcal{P}_{3}
$$

and

$$
w-y \leq \psi_{\bar{p}}(x, y) \leq w \quad \text { for } \quad(x, y) \in T, \bar{p} \in \mathcal{P}_{3} .
$$

Lemma 2.1. Let $(x, y) \in T$ and $\bar{p}=\left(p_{1}, p_{2}, p_{3}\right) \in \mathcal{P}_{3}$. Then: 
(i)

$$
\psi_{\bar{p}}(x, y)>0
$$

if and only if

$$
\left(g\left(p_{1}+p_{2}\right)-g\left(p_{1}\right)\right) u(y-x)+g\left(p_{1}\right) u(y)<u(w) ;
$$

(ii)

$$
\psi_{\bar{p}}(x, y) \leq 0 \leq \phi_{\bar{p}}(x, y)
$$

if and only if

$h\left(p_{3}\right) u(x-y)+g\left(p_{1}\right) u(x) \leq u(w) \leq\left(g\left(p_{1}+p_{2}\right)-g\left(p_{1}\right)\right) u(y-x)+g\left(p_{1}\right) u(y) ;$

(iii)

$$
\phi_{\bar{p}}(x, y)<0
$$

if and only if

$$
u(w)<h\left(p_{3}\right) u(x-y)+g\left(p_{1}\right) u(x) .
$$

Furthermore, the left (right) inequality in (21) is strict if and only if so is the right (left) inequality in (22).

Proof. Let $X:=\langle 0, x, y ; \bar{p}\rangle$ and let $d_{X}: \mathbb{R} \rightarrow \mathbb{R}$ be given by

$$
d_{X}(t)=E_{g h}[u(w+t-X)]-u(w) \text { for } t \in \mathbb{R} .
$$

Since the generalized Choquet integral is monotone and $u$ is strictly increasing, $d_{X}$ is non-decreasing. Furthermore, as $g(p), h(p) \in(0,1)$ for $p \in(0,1)$, applying [4, Lemma 3.3] we conclude that $d_{X}$ is injective. Hence, $d_{X}$ is strictly increasing. Moreover, we have $0<u(y-x)<u(y), u(x-y)<0<u(x)$,

$$
u(y-X)=\left\langle 0, u(y-x), u(y) ; p_{3}, p_{2}, p_{1}\right\rangle
$$

and

$$
u(x-X)=\left\langle u(x-y), 0, u(x) ; p_{3}, p_{2}, p_{1}\right\rangle .
$$

Thus, considering (10) and (11), we obtain

$d_{X}(y-w)=E_{g h}[u(y-X)]-u(w)=\left(g\left(p_{1}+p_{2}\right)-g\left(p_{1}\right)\right) u(y-x)+g\left(p_{1}\right) u(y)-u(w)$ and

$$
d_{X}(x-w)=E_{g h}[u(x-X)]-u(w)=h\left(p_{3}\right) u(x-y)+g\left(p_{1}\right) u(x)-u(w),
$$

respectively. On the other hand, considering (5), from (14) and (15) we derive that

$$
d_{X}\left(\phi_{\bar{p}}(x, y)+x-w\right)=d_{X}\left(\psi_{\bar{p}}(x, y)+y-w\right)=d_{X}\left(H_{(u, g, h)}(X)\right)=0 .
$$

Therefore, as $d_{X}$ is strictly increasing, we obtain (i)-(iii) as well as the second part of the assertion.

Corollary 2.2. Assume that $(x, y) \in T$ and $\bar{p}=\left(p_{1}, p_{2}, p_{3}\right) \in \mathcal{P}_{3}$. 
(i) If (20) holds, then

$$
\begin{gathered}
\left(1-g\left(p_{1}+p_{2}\right)\right) u\left(\psi_{\bar{p}}(x, y)\right)+\left(g\left(p_{1}+p_{2}\right)-g\left(p_{1}\right)\right) u\left(\phi_{\bar{p}}(x, y)\right) \\
+g\left(p_{1}\right) u\left(\phi_{\bar{p}}(x, y)+x\right)=u(w) .
\end{gathered}
$$

(ii) If (22) is valid, then

$$
\begin{gathered}
h\left(p_{3}\right) u\left(\psi_{\bar{p}}(x, y)\right)+\left(g\left(p_{1}+p_{2}\right)-g\left(p_{1}\right)\right) u\left(\phi_{\bar{p}}(x, y)\right) \\
+g\left(p_{1}\right) u\left(\phi_{\bar{p}}(x, y)+x\right)=u(w) .
\end{gathered}
$$

(iii) If (24) holds, then

$$
\begin{gathered}
h\left(p_{3}\right) u\left(\psi_{\bar{p}}(x, y)\right)+\left(h\left(p_{2}+p_{3}\right)-h\left(p_{3}\right)\right) u\left(\phi_{\bar{p}}(x, y)\right) \\
+g\left(p_{1}\right) u\left(\phi_{\bar{p}}(x, y)+x\right)=u(w) .
\end{gathered}
$$

Proof. Let $X:=\langle 0, x, y ; \bar{p}\rangle$. Then, in view of (14)-(15), we get

$$
w+H_{(u, g, h)}(X)-X=\left\langle\psi_{\bar{p}}(x, y), \phi_{\bar{p}}(x, y), \phi_{\bar{p}}(x, y)+x ; p_{3}, p_{2}, p_{1}\right\rangle .
$$

Moreover, as $w \in[0, \infty)$, from (8) and (14) we derive that $\phi_{\bar{p}}(x, y)+x=$ $w+H_{(u, g, h)}(X) \geq 0$. Therefore, making use of (5) and (10)-(12), in view of Lemma 2.1, we obtain assertions (i)-(iii).

Lemma 2.3. Assume that $w>0$ and put $L:=\lim _{x \rightarrow \infty} u(x)$.

(a) If $L=\infty$, then for every $\bar{p} \in \mathcal{P}_{3}$ there exists $(x, y) \in T$ such that

$$
\psi_{\bar{p}}(x, y)<0<\phi_{\bar{p}}(x, y) .
$$

(b) If $L<\infty$ and $\bar{p}=\left(p_{1}, p_{2}, p_{3}\right) \in \mathcal{P}_{3}$ is such that

$$
g\left(1-p_{3}\right)>u(w) / L
$$

then (28) is valid for some $(x, y) \in T$.

(c) If $L<\infty$ and (21) is satisfied for some $\bar{p}=\left(p_{1}, p_{2}, p_{3}\right) \in \mathcal{P}_{3}$ and $(x, y) \in$ $T$, then (29) holds.

Proof. (a) If $L=\infty$, then taking $x \in(0, w)$ and sufficiently big $x \in(0, w)$, we conclude that $(x, y) \in T$ and $(22)$ holds, with both inequalities being strict. Thus, according to Lemma 2.1(ii), (28) is valid for such $(x, y)$.

(b) Assume that $L<\infty$ and $\bar{p} \in \mathcal{P}_{3}$ is such that (29) holds. Then, for every $x \in(0, \infty)$, we have

$\lim _{y \rightarrow \infty}\left(\left(g\left(p_{1}+p_{2}\right)-g\left(p_{1}\right)\right) u(y-x)+g\left(p_{1}\right) u(y)\right)=g\left(p_{1}+p_{2}\right) L=g\left(1-p_{3}\right) L>u(w)$.

Thus, for every $x \in(0, w)$ and sufficiently big $y \in(w, \infty),(22)$ is valid, with both inequalities being strict. Hence, applying Lemma 2.1(ii), we conclude that (28) holds for some $(x, y) \in T$. 
(c) Assume that $L<\infty$ and (21) holds for some $\bar{p}=\left(p_{1}, p_{2}, p_{3}\right) \in \mathcal{P}_{3}$ and $(x, y) \in T$. Then, according to Lemma 2.1(ii), (22) is satisfied and so $u(w) \leq\left(g\left(p_{1}+p_{2}\right)-g\left(p_{1}\right)\right) u(y-x)+g\left(p_{1}\right) u(y) \leq g\left(p_{1}+p_{2}\right) u(y)<g\left(1-p_{3}\right) L$. Thus, (29) is valid.

Lemma 2.4. For every $\bar{p} \in \mathcal{P}_{3}$, the functions $\phi_{\bar{p}}$ and $\psi_{\bar{p}}$ are continuous.

Proof. Let $\bar{p} \in \mathcal{P}_{3}$. In view of (16), it is enough to show that $\phi_{\bar{p}}$ is continuous. Furthermore, it follows from (9) and (14) that $\phi_{\bar{p}}$ is monotone in $y$ for each $x$. Thus, in order to prove the continuity of $\phi_{\bar{p}}$, it suffices to show that it is continuous in $x$ and $y$ separately. Since in both cases similar arguments work, we prove only the continuity of $\phi_{\bar{p}}$ in $x$. Suppose that, for some $y \in$ $(0, \infty), \phi_{\bar{p}}(\cdot, y)$ is not continuous at the point $x \in(0, y)$. Then there exists a sequence $\left(x_{n}: n \in \mathbb{N}\right)$ of elements of $(0, y)$ such that $\lim _{n \rightarrow \infty} x_{n}=x$ but $\left(\phi_{\bar{p}}\left(x_{n}, y\right): n \in \mathbb{N}\right)$ does not tend to $\phi_{\bar{p}}(x, y)$. According to (17), the sequence $\left(\phi_{\bar{p}}\left(x_{n}, y\right): n \in \mathbb{N}\right)$ is bounded, so there exists a subsequence $\left(x_{n_{k}}: k \in \mathbb{N}\right)$ of the sequence $\left(x_{n}: n \in \mathbb{N}\right)$ such that $\lim _{k \rightarrow \infty} \phi_{\bar{p}}\left(x_{n_{k}}, y\right)=: d \neq \phi_{\bar{p}}(x, y)$. Note that, in view of (16), we have

$$
\lim _{k \rightarrow \infty} \psi_{\bar{p}}\left(x_{n_{k}}, y\right)=d+x-y .
$$

Moreover, the following three cases are possible:

1. $\psi_{\bar{p}}(x, y) \geq 0$,

2. $\psi_{\bar{p}}(x, y)<0<\phi_{\bar{p}}(x, y)$,

3. $\phi_{\bar{p}}(x, y) \leq 0$.

Case 1 If $\psi_{\bar{p}}(x, y)>0$, then in view of Lemma 2.1(i), (20) is valid and so, for sufficiently big $k \in \mathbb{N}$, we get

$$
\left(g\left(p_{1}+p_{2}\right)-g\left(p_{1}\right)\right) u\left(y-x_{n_{k}}\right)+g\left(p_{1}\right) u(y)<u(w) .
$$

Furthermore, applying Corollary 2.2(i), we obtain (25) and

$$
\begin{gathered}
\left(1-g\left(p_{1}+p_{2}\right)\right) u\left(\psi_{\bar{p}}\left(x_{n_{k}}, y\right)\right)+\left(g\left(p_{1}+p_{2}\right)-g\left(p_{1}\right)\right) u\left(\phi_{\bar{p}}\left(x_{n_{k}}, y\right)\right) \\
+g\left(p_{1}\right) u\left(\phi_{\bar{p}}\left(x_{n_{k}}, y\right)+x_{n_{k}}\right)=u(w)
\end{gathered}
$$

for sufficiently big $k \in \mathbb{N}$. Letting $k \rightarrow \infty$ in (31) and subtracting the equality obtained this way from (25), in view of (30), we get

$$
\begin{gathered}
\left(1-g\left(p_{1}+p_{2}\right)\right)\left(u\left(\psi_{\bar{p}}(x, y)\right)-u(d+x-y)\right) \\
+\left(g\left(p_{1}+p_{2}\right)-g\left(p_{1}\right)\right)\left(u\left(\phi_{\bar{p}}(x, y)\right)-u(d)\right) \\
+g\left(p_{1}\right)\left(u\left(\phi_{\bar{p}}(x, y)+x\right)-u(d+x)\right)=0 .
\end{gathered}
$$

On the other hand $g$ is non-decreasing, with $g(p) \in(0,1)$ for $p \in(0,1)$, and $u$ is strictly increasing. Thus, making use of (16), we conclude that the left hand 
side of the last equality is positive whenever $d<\phi_{\bar{p}}(x, y)$, and it is negative whenever $d>\phi_{\bar{p}}(x, y)$. This yields a contradiction.

Suppose that $\psi_{\bar{p}}(x, y)=0$. Then, in view of $(16), \phi_{\bar{p}}(x, y)=y-x>0$ and so, applying Lemma 2.1(i) and Corollary 2.2(i), we obtain

$$
h\left(p_{3}\right) u(x-y)+g\left(p_{1}\right) u(x)<u(w)
$$

and

$$
\left(g\left(p_{1}+p_{2}\right)-g\left(p_{1}\right)\right) u(y-x)+g\left(p_{1}\right) u(y)=u(w) .
$$

If $d>y-x$, then making use of (30), we get $\lim _{k \rightarrow \infty} \psi_{\bar{p}}\left(x_{n_{k}}, y\right)>0$. Hence, for sufficiently big $k \in \mathbb{N}$, we have $\psi_{\bar{p}}\left(x_{n_{k}}, y\right)>0$ and so, according to Lemma 2.1(i) and Corollary 2.2(i), (31) holds. Thus, passing in (31) to the limit as $k \rightarrow \infty$ and subtracting the obtained equality from (33), in view of (30), we get

$$
\begin{gathered}
\left(g\left(p_{1}+p_{2}\right)-g\left(p_{1}\right)\right)(u(y-x)-u(d)) \\
+g\left(p_{1}\right)(u(y)-u(d+x))-\left(1-g\left(p_{1}+p_{2}\right)\right) u(d+x-y)=0 .
\end{gathered}
$$

However, as $u$ is strictly increasing and $g$ is non-decreasing, with $g(p) \in(0,1)$ for $p \in(0,1)$, the left hand side of this equality is negative, which gives a contradiction.

If $d<y-x$, then in view of $(30)$, we get $\lim _{k \rightarrow \infty} \psi_{\bar{p}}\left(x_{n_{k}}, y\right)<0$. Thus $\psi_{\bar{p}}\left(x_{n_{k}}, y\right)<0$ for sufficiently big $k \in \mathbb{N}$. On the other hand, making use of (32), for sufficiently big $k \in \mathbb{N}$, we get

$$
h\left(p_{3}\right) u\left(x_{n_{k}}-y\right)+g\left(p_{1}\right) u\left(x_{n_{k}}\right)<u(w)
$$

whence, by Lemma 2.1(ii), $\phi_{\bar{p}}\left(x_{n_{k}}, y\right)>0$. Therefore, applying Lemma 2.1(ii) and Corollary 2.2(ii), we get

$$
\begin{gathered}
h\left(p_{3}\right) u\left(\psi_{\bar{p}}\left(x_{n_{k}}, y\right)\right)+\left(g\left(p_{1}+p_{2}\right)-g\left(p_{1}\right)\right) u\left(\phi_{\bar{p}}\left(x_{n_{k}}, y\right)\right) \\
+g\left(p_{1}\right) u\left(\phi_{\bar{p}}\left(x_{n_{k}}, y\right)+x_{n_{k}}\right)=u(w)
\end{gathered}
$$

for sufficiently big $k \in \mathbb{N}$. Letting in the last equality $k \rightarrow \infty$ and subtracting the obtained equality from (33), in view of (30), we obtain

$$
\begin{gathered}
\left(g\left(p_{1}+p_{2}\right)-g\left(p_{1}\right)\right)(u(y-x)-u(d)) \\
+g\left(p_{1}\right)(u(y)-u(d+x))-h\left(p_{3}\right) u(d+x-y)=0 .
\end{gathered}
$$

Since $d<y-x$, arguing as previously, we conclude that the left hand side of this equality is positive, which yields a contradiction.

Case 2 Applying Lemma 2.1(ii) and Corollary 2.2(ii), we obtain (22), with both inequalities being strict, and (26). Hence, we have $h\left(p_{3}\right) u\left(x_{n_{k}}-y\right)+g\left(p_{1}\right) u\left(x_{n_{k}}\right)<u(w)<\left(g\left(p_{1}+p_{2}\right)-g\left(p_{1}\right)\right) u\left(y-x_{n_{k}}\right)+g\left(p_{1}\right) u(y)$ 
for sufficiently big $k \in \mathbb{N}$. Therefore, according to Corollary 2.2(ii), for sufficiently big $k \in \mathbb{N}$, we get

$$
\begin{gathered}
h\left(p_{3}\right) u\left(\psi_{\bar{p}}\left(x_{n_{k}}, y\right)\right)+\left(g\left(p_{1}+p_{2}\right)-g\left(p_{1}\right)\right) u\left(\phi_{\bar{p}}\left(x_{n_{k}}, y\right)\right) \\
+g\left(p_{1}\right) u\left(\phi_{\bar{p}}\left(x_{n_{k}}, y\right)+x_{n_{k}}\right)=u(w) .
\end{gathered}
$$

Thus, making use of (26) and arguing as in the previous case, we obtain

$$
\begin{aligned}
h\left(p_{3}\right)\left(u\left(\psi_{\bar{p}}(x, y)\right)\right. & -u(d+x-y))+\left(g\left(p_{1}+p_{2}\right)-g\left(p_{1}\right)\right)\left(u\left(\phi_{\bar{p}}(x, y)\right)-u(d)\right) \\
& +g\left(p_{1}\right)\left(u\left(\phi_{\bar{p}}(x, y)+x\right)-u(d+x)\right)=0,
\end{aligned}
$$

which gives a contradiction.

Case 3 If $\phi_{\bar{p}}(x, y)<0$, then according to Lemma 2.1(iii), we have (24). Thus

$$
u(w)<h\left(p_{3}\right) u\left(x_{n_{k}}-y\right)+g\left(p_{1}\right) u\left(x_{n_{k}}\right)
$$

for sufficiently big $k \in \mathbb{N}$. Furthermore, applying Corollary 2.2(iii), we obtain (27) and

$$
\begin{gathered}
h\left(p_{3}\right) u\left(\psi_{\bar{p}}\left(x_{n_{k}}, y\right)\right)+\left(h\left(p_{2}+p_{3}\right)-h\left(p_{3}\right)\right) u\left(\phi_{\bar{p}}\left(x_{n_{k}}, y\right)\right) \\
+g\left(p_{1}\right) u\left(\phi_{\bar{p}}\left(x_{n_{k}}, y\right)+x_{n_{k}}\right)=u(w)
\end{gathered}
$$

for sufficiently big $k \in \mathbb{N}$. Hence, repeating the arguments from the first case, we get

$$
\begin{aligned}
h\left(p_{3}\right)\left(u\left(\psi_{\bar{p}}(x, y)\right)\right. & -u(d+x-y))+\left(h\left(p_{2}+p_{3}\right)-h\left(p_{3}\right)\right)\left(u\left(\phi_{\bar{p}}(x, y)\right)-u(d)\right) \\
& +g\left(p_{1}\right)\left(u\left(\phi_{\bar{p}}(x, y)+x\right)-u(d+x)\right)=0,
\end{aligned}
$$

which yields a contradiction.

If $\phi_{\bar{p}}(x, y)=0$, then in view of (16), $\psi_{\bar{p}}(x, y)=x-y<0$. Hence, according to Lemma 2.1(ii) and Corollary 2.2(ii), we have

$$
u(w)<\left(g\left(p_{1}+p_{2}\right)-g\left(p_{1}\right)\right) u(y-x)+g\left(p_{1}\right) u(y)
$$

and

$$
h\left(p_{3}\right) u(x-y)+g\left(p_{1}\right) u(x)=u(w) .
$$

It follows from (35) that, for sufficiently big $k \in \mathbb{N}$,

$$
u(w)<\left(g\left(p_{1}+p_{2}\right)-g\left(p_{1}\right)\right) u\left(y-x_{n_{k}}\right)+g\left(p_{1}\right) u(y)
$$

whence, in view of Lemma 2.1(iii), $\psi_{\bar{p}}\left(x_{n_{k}}, y\right)<0$. Therefore, if $d>0$, then for sufficiently big $k \in \mathbb{N}$, we have $\psi_{\bar{p}}\left(x_{n_{k}}, y\right)<0<\phi_{\bar{p}}\left(x_{n_{k}}, y\right)$ and so, according to Lemma 2.1(ii) and Corollary 2.2(ii), (34) holds. Passing to the limit in (34) 
as $k \rightarrow \infty$ and subtracting the equality obtained this way from (36), in view of $(30)$, we get

$$
\begin{gathered}
h\left(p_{3}\right)(u(x-y)-u(d+x-y))+g\left(p_{1}\right)(u(x)-u(d+x)) \\
-\left(g\left(p_{1}+p_{2}\right)-g\left(p_{1}\right)\right) u(d)=0 .
\end{gathered}
$$

Since $d>0$, the left hand side of this equality is negative and so we get a contradiction.

If $d<0$, then $\phi_{\bar{p}}\left(x_{n_{k}}, y\right)<0$ for sufficiently big $k \in \mathbb{N}$. Thus, applying Lemma 2.1(iii) and Corollary 2.2(iii), we conclude that

$$
\begin{gathered}
h\left(p_{3}\right) u\left(\psi_{\bar{p}}\left(x_{n_{k}}, y\right)\right)+\left(h\left(p_{2}+p_{3}\right)-h\left(p_{3}\right)\right) u\left(\phi_{\bar{p}}\left(x_{n_{k}}, y\right)\right) \\
+g\left(p_{1}\right) u\left(\phi_{\bar{p}}\left(x_{n_{k}}, y\right)+x_{n_{k}}\right)=u(w)
\end{gathered}
$$

for sufficiently big $k \in \mathbb{N}$. Hence, arguing as previously, we obtain

$$
\begin{gathered}
h\left(p_{3}\right)(u(x-y)-u(d+x-y))+g\left(p_{1}\right)(u(x)-u(d+x)) \\
-\left(h\left(p_{2}+p_{3}\right)-h\left(p_{3}\right)\right) u(d)=0 .
\end{gathered}
$$

However, as $d<0$, the left hand side of the last equality is positive. So, we have a contradiction.

This proves the continuity of $\phi_{\bar{p}}(\cdot, y)$ at $x$.

Lemma 2.5. Let $\bar{p} \in \mathcal{P}_{3}$.

(a) If $w=0$, then for every $y \in(0, \infty), 0$ is an interior point of $\phi_{\bar{p}}((0, y) \times$ $\{y\})$.

(b) If $w>0$, then for every $y \in(0, w), w$ is an interior point of $\phi_{\bar{p}}((0, y) \times$ $\{y\})$.

Proof. Note that, for every $y \in(0, \infty)$, we have

$$
\begin{aligned}
& \lim _{x \rightarrow y^{-}}\left(h\left(p_{3}\right) u(x-y)+g\left(p_{1}\right) u(x)\right)=g\left(p_{1}\right) u(y), \\
& \lim _{x \rightarrow 0^{+}}\left(h\left(p_{3}\right) u(x-y)+g\left(p_{1}\right) u(x)\right)=h\left(p_{3}\right) u(-y)
\end{aligned}
$$

and

$$
\lim _{x \rightarrow 0^{+}}\left(\left(g\left(p_{1}+p_{2}\right)-g\left(p_{1}\right)\right) u(y-x)+g\left(p_{1}\right) u(y)\right)=g\left(p_{1}+p_{2}\right) u(y) .
$$

Therefore, if $w=0$, then taking an arbitrary $y \in(0, \infty)$, from (37) we derive that, for $x \in(0, y)$ sufficiently close to $y,(24)$ is valid. Thus, according to Lemma 2.1(iii), (23) holds for some $x \in(0, y)$. Furthermore, as $u$ is strictly increasing, making use of (38) and (39), we obtain that, for $x \in(0, y)$ sufficiently close to $0,(22)$ is satisfied, with both inequalities being strict. So, applying Lemma 2.1(ii), we conclude that $\phi_{\bar{p}}(x, y)>0$ for some $x \in(0, y)$. Hence, as $\phi_{\bar{p}}$ is continuous, 0 belongs to the interior of $\phi_{\bar{p}}((0, y) \times\{y\})$. 
Now assume that $w>0$ and fix $y \in(0, w)$. Then, for every $x \in(0, y)$, (20) is satisfied and so, according to Corollary 2.2(i), (25) holds. Suppose that $\phi_{\bar{p}}(x, y) \geq w$ for $x \in(0, y)$. Then, in view of (16) and (17), we get

$$
\lim _{x \rightarrow y^{-}} \phi_{\bar{p}}(x, y)=\lim _{x \rightarrow y^{-}} \psi_{\bar{p}}(x, y)=w .
$$

Hence, letting in (25) $x \rightarrow y^{-}$, we obtain $g\left(p_{1}\right)(u(w+y)-u(w))=0$. Since $u$ is strictly increasing and $g\left(p_{1}\right)>0$, this yields a contradiction. In this way we have proved that $\phi_{\bar{p}}(x, y)<w$ for some $x \in(0, y)$. On the other hand, if $\phi_{\bar{p}}(x, y) \leq w$ for $x \in(0, y)$, then applying (17), we get $\lim _{x \rightarrow 0^{+}} \phi_{\bar{p}}(x, y)=w$. Thus, in view of (16), we have $\lim _{x \rightarrow 0^{+}} \psi_{\bar{p}}(x, y)=w-y$ and so, letting in (25) $x \rightarrow 0^{+}$, we obtain $\left(1-g\left(p_{1}+p_{2}\right)\right)(u(w-y)-u(w))=0$. Since $u$ is strictly increasing and $g\left(p_{1}+p_{2}\right)<1$, this gives a contradiction. Therefore $\phi_{\bar{p}}(x, y)>w$ for some $x \in(0, y)$ and so, as $\phi_{\bar{p}}$ is continuous, $w$ is an interior point of $\phi_{\bar{p}}((0, y) \times\{y\})$.

Lemma 2.6. A family $\left\{\phi_{\bar{p}}(T): \bar{p} \in \mathcal{P}_{3}\right\}$ is a cover of $[w, \infty)$ and a family $\left\{\psi_{\bar{p}}(T): \bar{p} \in \mathcal{P}_{3}\right\}$ is a cover of $(-\infty, w)$, that is

$$
[w, \infty) \subset \bigcup_{\bar{p} \in \mathcal{P}_{3}} \phi_{\bar{p}}(T)
$$

and

$$
(-\infty, w) \subset \bigcup_{\bar{p} \in \mathcal{P}_{3}} \psi_{\bar{p}}(T),
$$

respectively.

Proof. In order to prove (40), fix $(x, y) \in T$. Since $g$ and $h$ are continuous, with $g(0)=0$ and $h(1)=1$, in the case where $w=0$, for sufficiently small $p_{1}, p_{2} \in(0,1)$, we have

$h\left(1-p_{1}-p_{2}\right) u(x-y)+g\left(p_{1}\right) u(x)<u(w)<\left(g\left(p_{1}+p_{2}\right)-g\left(p_{1}\right)\right) u(y-x)+g\left(p_{1}\right) u(y)$.

Hence, applying Corollary 2.2(ii), we obtain

$$
\begin{gathered}
h\left(1-p_{1}-p_{2}\right) u\left(\psi_{\left(p_{1}, p_{2}, 1-p_{1}-p_{2}\right)}(x, y)\right) \\
+\left(g\left(p_{1}+p_{2}\right)-g\left(p_{1}\right)\right) u\left(\phi_{\left(p_{1}, p_{2}, 1-p_{1}-p_{2}\right)}(x, y)\right) \\
+g\left(p_{1}\right) u\left(\phi_{\left(p_{1}, p_{2}, 1-p_{1}-p_{2}\right)}(x, y)+x\right)=u(w)
\end{gathered}
$$

for sufficiently small $p_{1}, p_{2} \in(0,1)$. Thus, taking into account (17) and (18), we get

$$
\lim _{p_{1}, p_{2} \rightarrow 0^{+}} u\left(\psi_{\left(p_{1}, p_{2}, 1-p_{1}-p_{2}\right)}(x, y)\right)=u(w) .
$$

Since $u$ is a continuous, strictly increasing function, this implies that

$$
\lim _{p_{1}, p_{2} \rightarrow 0^{+}} \psi_{\left(p_{1}, p_{2}, 1-p_{1}-p_{2}\right)}(x, y)=w .
$$


If $w>0$, then for sufficiently small $p_{1}, p_{2} \in(0,1),(20)$ holds and so, applying Corollary 2.2(i), we get

$$
\begin{gathered}
\left(1-g\left(p_{1}+p_{2}\right)\right) u\left(\psi_{\left(p_{1}, p_{2}, 1-p_{1}-p_{2}\right)}(x, y)\right) \\
+\left(g\left(p_{1}+p_{2}\right)-g\left(p_{1}\right)\right) u\left(\phi_{\left(p_{1}, p_{2}, 1-p_{1}-p_{2}\right)}(x, y)\right) \\
+g\left(p_{1}\right) u\left(\phi_{\left(p_{1}, p_{2}, 1-p_{1}-p_{2}\right)}(x, y)+x\right)=u(w) .
\end{gathered}
$$

Thus, repeating the previous arguments, we obtain (42). In this way we have proved that (42) holds for every $(x, y) \in T$. Therefore, taking $x_{0} \in[w, \infty)$ and $(x, y) \in T$, with $y-x>x_{0}-w$, in view of $(16)$, we get

$$
\begin{aligned}
\lim _{p_{1}, p_{2} \rightarrow 0^{+}} \phi_{\left(1-p_{1}-p_{2}, p_{1}, p_{2}\right)}(x, y) & =\lim _{p_{1}, p_{2} \rightarrow 0^{+}}\left(\psi_{\left(1-p_{1}-p_{2}, p_{1}, p_{2}\right)}(x, y)+y-x\right) \\
& =w+y-x>x_{0} .
\end{aligned}
$$

Hence, there exists $\bar{p} \in \mathcal{P}_{3}$ such that $\phi_{\bar{p}}(x, y)>x_{0}$. On the other hand, from Lemma 2.4 and Lemma 2.5 we deduce that $\phi_{\bar{p}}(T)$ is connected and $w$ is its interior point, respectively. Thus $x_{0} \in \phi_{\bar{p}}(T)$, which proves (40).

Now, we show that (41) holds. To this end, fix $x_{0} \in(-\infty, w)$. Let $(x, y) \in T$ be such that $x<w$ and $y>\max \left\{w, w-x_{0}\right\}$. Then, for sufficiently small $p_{2}, p_{3} \in(0,1)$, we have

$$
\begin{gathered}
h\left(p_{3}\right) u(x-y)+g\left(1-p_{2}-p_{3}\right) u(x)<u(w) \\
<\left(g\left(1-p_{3}\right)-g\left(1-p_{2}-p_{3}\right)\right) u(y-x)+g\left(1-p_{2}-p_{3}\right) u(y)
\end{gathered}
$$

and so, applying Corollary 2.2(ii), we obtain

$$
\begin{gathered}
h\left(p_{3}\right) u\left(\psi_{\left(1-p_{2}-p_{3}, p_{2}, p_{3}\right)}(x, y)\right)+\left(g\left(1-p_{3}\right)-g\left(1-p_{2}-p_{3}\right)\right) u\left(\phi_{\left(1-p_{2}-p_{3}, p_{2}, p_{3}\right)}(x, y)\right) \\
+g\left(1-p_{2}-p_{3}\right) u\left(\phi_{\left(1-p_{2}-p_{3}, p_{2}, p_{3}\right)}(x, y)+x\right)=u(w) .
\end{gathered}
$$

Thus, arguing as previously, we get

$$
\lim _{p_{2}, p_{3} \rightarrow 0^{+}} u\left(\phi_{\left(1-p_{2}-p_{3}, p_{2}, p_{3}\right)}(x, y)+x\right)=u(w)
$$

and so

$$
\lim _{p_{2}, p_{3} \rightarrow 0^{+}} \phi_{\left(1-p_{2}-p_{3}, p_{2}, p_{3}\right)}(x, y)=w-x .
$$

Hence, considering (16), we obtain

$$
\lim _{p_{2}, p_{3} \rightarrow 0^{+}} \psi_{\left(1-p_{2}-p_{3}, p_{2}, p_{3}\right)}(x, y)=w-y<x_{0} .
$$

So, there exists $\bar{p} \in \mathcal{P}_{3}$ such that $\psi_{\bar{p}}(x, y)<x_{0}$. Furthermore, in view of (18), we get $\lim _{y \rightarrow 0^{+}} \psi_{\bar{p}}(y / 2, y)=w$. Since, in view of Lemma 2.4, $\psi_{\bar{p}}(T)$ is connected, this means that $x_{0} \in \phi_{\bar{p}}(T)$ and so, (41) is proved. 
Lemma 2.7. Let $\bar{p}=\left(p_{1}, p_{2}, p_{3}\right) \in \mathcal{P}_{3}$ and $F_{\bar{p}}: T \rightarrow \mathbb{R}^{2}$ be defined as follows

$$
F_{\bar{p}}(x, y)=\left(\phi_{\bar{p}}(x, y), \psi_{\bar{p}}(x, y)\right) \quad \text { for } \quad(x, y) \in T .
$$

Then $F_{\bar{p}}(T)$ is open.

Proof. It follows from Lemma 2.4 that $F_{\bar{p}}$ is continuous. We show that it is injective. Suppose that $F_{\bar{p}}\left(x_{1}, y_{1}\right)=F_{\bar{p}}\left(x_{2}, y_{2}\right)$ for some $\left(x_{1}, y_{1}\right),\left(x_{2}, y_{2}\right) \in T$. Then

$$
\phi_{\bar{p}}\left(x_{1}, y_{1}\right)=\phi_{\bar{p}}\left(x_{2}, y_{2}\right)
$$

and

$$
\psi_{\bar{p}}\left(x_{1}, y_{1}\right)=\psi_{\bar{p}}\left(x_{2}, y_{2}\right) .
$$

Therefore, applying Lemma 2.1 and Corollary 2.2, we obtain

$$
\begin{aligned}
& g\left(p_{1}\right) u\left(\phi_{\bar{p}}\left(x_{1}, y_{1}\right)+x_{1}\right)=u(w)-\gamma u\left(\phi_{\bar{p}}\left(x_{1}, y_{1}\right)\right)-\delta u\left(\psi_{\bar{p}}\left(x_{1}, y_{1}\right)\right) \\
& \quad=u(w)-\gamma u\left(\phi_{\bar{p}}\left(x_{2}, y_{2}\right)\right)-\delta u\left(\psi_{\bar{p}}\left(x_{2}, y_{2}\right)\right)=g\left(p_{1}\right) u\left(\phi_{\bar{p}}\left(x_{2}, y_{2}\right)+x_{2}\right),
\end{aligned}
$$

where

$$
\gamma:= \begin{cases}g\left(p_{1}+p_{2}\right)-g\left(p_{1}\right) & \text { whenever } \quad \phi_{\bar{p}}\left(x_{1}, y_{1}\right) \geq 0 \\ h\left(p_{2}+p_{3}\right)-h\left(p_{3}\right) & \text { otherwise }\end{cases}
$$

and

$$
\delta:=\left\{\begin{array}{ll}
1-g\left(p_{1}+p_{2}\right) & \text { whenever } \\
h\left(p_{3}\right) & \text { otherwise. }
\end{array} \psi_{\bar{p}}\left(x_{1}, y_{1}\right)>0,\right.
$$

Thus, as $u$ is strictly increasing and $g\left(p_{1}\right)>0$, we get

$$
\phi_{\bar{p}}\left(x_{1}, y_{1}\right)+x_{1}=\phi_{\bar{p}}\left(x_{2}, y_{2}\right)+x_{2} .
$$

Hence, in view of (44), we obtain $x_{1}=x_{2}$. Furthermore, considering (16), (44) and (45), we conclude that

$$
y_{1}=\phi_{\bar{p}}\left(x_{1}, y_{1}\right)-\psi_{\bar{p}}\left(x_{1}, y_{1}\right)+x_{1}=\phi_{\bar{p}}\left(x_{2}, y_{2}\right)-\psi_{\bar{p}}\left(x_{2}, y_{2}\right)+x_{2}=y_{2},
$$

which completes the proof of the injectivity of $F_{\bar{p}}$.

Now, as $F_{\bar{p}}$ is continuous and injective, applying the Invariant Domain Theorem, we obtain that $F_{\bar{p}}(T)$ is open.

The following lemma, concerning the solutions of the general linear equation on a region, will play an important role in the proof of our main results. For more details concerning the general linear equation we refer to [11, Chapter $13.10]$.

Lemma 2.8. Assume that $D$ is a nonempty, open and connected subset of $\mathbb{R}^{2}$, $f: \mathbb{R} \rightarrow \mathbb{R}$ is a nonconstant continuous function, $a, b, A, B \in \mathbb{R} \backslash\{0\}$ and $c, C \in \mathbb{R}$. Then $f$ satisfies the equation

$$
f(a x+b y+c)=A f(x)+B f(y)+C \quad \text { for } \quad(x, y) \in D
$$


if and only if there exist $\gamma \in \mathbb{R} \backslash\{0\}$ and $\beta_{1}, \beta_{2} \in \mathbb{R}$ such that

$$
\begin{aligned}
& f(x)=\frac{\gamma a}{A} x+\beta_{1} \quad \text { for } \quad x \in D_{1}, \\
& f(x)=\frac{\gamma b}{B} x+\beta_{2} \quad \text { for } \quad x \in D_{2}
\end{aligned}
$$

and

$$
f(x)=\gamma(x-c)+A \beta_{1}+B \beta_{2}+C \text { for } x \in\{a s+b t+c:(s, t) \in D\},
$$

where

$$
D_{1}:=\{x \in \mathbb{R}:(x, y) \in D \text { for some } y \in \mathbb{R}\}
$$

and

$$
D_{2}:=\{y \in \mathbb{R}:(x, y) \in D \text { for some } x \in \mathbb{R}\} .
$$

Proof. Assume that $f$ satisfies (46). Replacing in (46) $x$ and $y$ by $x / a$ and $y / b$, respectively, we conclude that a triple of functions $\left(f_{1}, f_{2}, f_{3}\right)$, where $f_{1}(x)=$ $A f(x / a)$ for $x \in \mathbb{R}, f_{2}(x)=B f(x / b)$ for $x \in \mathbb{R}$ and $f_{3}(x)=f(x+c)-C$ for $x \in \mathbb{R}$, satisfies the Pexider equation

$$
f_{3}(x+y)=f_{1}(x)+f_{2}(y) \text { for }(x, y) \in(a, b) \cdot D,
$$

where $(a, b) \cdot D:=\{(a s, b t):(s, t) \in D\}$. Moreover, $f_{i}$ for $i \in\{1,2,3\}$ are nonconstant continuous functions and, as $D$ is nonempty, open and connected, so is $(a, b) \cdot D$. Therefore, applying [11, Theorem 13.3.5] and [13, Theorem 1], we obtain that there exist $\gamma \in \mathbb{R} \backslash\{0\}$ and $\delta_{1}, \delta_{2} \in \mathbb{R}$ such that

$$
\begin{aligned}
& f_{1}(x)=\gamma x+\delta_{1} \quad \text { for } \quad x \in a \cdot D_{1}:=\left\{a s: s \in D_{1}\right\}, \\
& f_{2}(x)=\gamma x+\delta_{2} \text { for } \quad x \in b \cdot D_{2}:=\left\{b t: t \in D_{2}\right\}
\end{aligned}
$$

and

$$
f_{3}(x)=\gamma x+\delta_{1}+\delta_{2} \quad \text { for } \quad x \in\{a s+b t:(s, t) \in D\} .
$$

Hence, considering the definitions of $f_{1}, f_{2}$ and $f_{3}$, we get (47)-(49) with $\beta_{1}:=\delta_{1} / A$ and $\beta_{2}:=\delta_{2} / B$.

The converse is easy to check.

We complete this section with one more result, which will be useful in our further considerations.

Lemma 2.9. Let $I \subseteq \mathbb{R}$ be an open interval and let $u_{1}, u_{2}: \mathbb{R} \rightarrow \mathbb{R}$ be strictly increasing functions. Assume that, for every $x \in I$, there exist $\alpha_{x} \in \mathbb{R} \backslash\{0\}$, $\beta_{x} \in \mathbb{R}$ and $r_{x} \in(0, \infty)$ such that

$$
u_{2}(z)=\alpha_{x} u_{1}(z)+\beta_{x} \text { for } z \in U_{x}:=\left(x-r_{x}, x+r_{x}\right) .
$$

Then there exist $\alpha \in \mathbb{R} \backslash\{0\}$ and $\beta \in \mathbb{R}$ such that

$$
u_{2}(z)=\alpha u_{1}(z)+\beta \text { for } z \in I .
$$


Proof. It is enough to show that, for every $x, y \in I$, we have $\alpha_{x}=\alpha_{y}$ and $\beta_{x}=\beta_{y}$. To this end, fix $x, y \in I$. Let $z_{x} \in U_{x}$ and $z_{y} \in U_{y}$. According to [6, Lemma 2.4], there exist $n \in \mathbb{N}$ and $x_{0}, x_{1}, \ldots, x_{n} \in I$ such that $z_{x} \in U_{x_{0}}$, $z_{y} \in U_{x_{n}}$ and $U_{x_{i-1}} \cap U_{x_{i}} \neq \emptyset$ for $i \in\{1, \ldots, n\}$. Thus, in view of (50), we get

$$
\alpha_{x} u_{1}(z)+\beta_{x}=\alpha_{x_{0}} u_{1}(z)+\beta_{x_{0}} \text { for } z \in U_{x} \cap U_{x_{0}} .
$$

Hence, if $\alpha_{x}$ and $\alpha_{x_{0}}$ were different, then we would have

$$
u_{1}(z)=\frac{\beta_{x_{0}}-\beta_{x}}{\alpha_{x}-\alpha_{x_{0}}} \text { for } z \in U_{x} \cap U_{x_{0}}
$$

which is not possible, as $U_{x} \cap U_{x_{0}}$ is a nonempty open set and $u_{1}$ is strictly increasing. Thus $\alpha_{x_{0}}=\alpha_{x}$ and so, in view of (52), $\beta_{x_{0}}=\beta_{x}$. Then, we have

$$
\alpha_{x_{0}} u_{1}(z)+\beta_{x_{0}}=\alpha_{x_{1}} u_{1}(z)+\beta_{x_{1}} \text { for } z \in U_{x_{0}} \cap U_{x_{1}} .
$$

Hence, arguing as previously, we obtain that $\alpha_{x_{0}}=\alpha_{x_{1}}$ and $\beta_{x_{0}}=\beta_{x_{1}}$. Therefore, $\alpha_{x}=\alpha_{x_{1}}$ and $\beta_{x}=\beta_{x_{1}}$. Repeating this procedure, we conclude finally that $\alpha_{x}=\alpha_{y}$ and $\beta_{x}=\beta_{y}$.

\section{Main results}

The following three theorems, concerning the extension problem for functionals of equivalent utility under the Cumulative Prospect Theory, are the main results of the paper.

Theorem 3.1. Let $w \in[0, \infty)$. Assume that, for $i \in\{1,2\}, u_{i}: \mathbb{R} \rightarrow \mathbb{R}$ is a strictly increasing continuous function with $u_{i}(0)=0$ and $g_{i}, h_{i}:[0,1] \rightarrow[0,1]$ are strictly increasing continuous probability distortion functions. Furthermore, assume that $H_{\left(u_{1}, g_{1}, h_{1}\right)}$ and $H_{\left(u_{2}, g_{2}, h_{2}\right)}$ are the functionals of equivalent utility, defined by (5), such that

$$
H_{\left(u_{1}, g_{1}, h_{1}\right)}(X)=H_{\left(u_{2}, g_{2}, h_{2}\right)}(X) \text { for } X \in \mathcal{X}_{0}^{(3)} .
$$

(a) If $w=0$ or $\lim _{x \rightarrow \infty} u_{1}(x)=\infty$, then $g_{1}=g_{2}, h_{1}=h_{2}$ and there exists $\alpha \in(0, \infty)$ such that

$$
u_{2}(x)=\alpha u_{1}(x) \text { for } x \in \mathbb{R} .
$$

(b) If $w>0$ and $L:=\lim _{x \rightarrow \infty} u_{1}(x)<\infty$, then $g_{1}=g_{2}$ and there exist $\alpha, \beta \in(0, \infty)$ such that

$$
h_{2}(p)=\frac{\beta}{\alpha} h_{1}(p) \quad \text { for } \quad p \in\left[0,1-g_{1}^{-1}\left(u_{1}(w) / L\right)\right]
$$

and

$$
u_{2}(x)=\left\{\begin{array}{lll}
\alpha u_{1}(x) & \text { for } & x \in(-\infty, 0) \\
\beta u_{1}(x) & \text { for } & x \in[0, \infty)
\end{array}\right.
$$


Proof. Let $T$ be given by (13) and let, for every $\bar{p} \in \mathcal{P}_{3}$ and $i \in\{1,2\}$, $\phi_{\bar{p}}^{(i)}, \psi_{\bar{p}}^{(i)}: T \rightarrow \mathbb{R}$ be defined as follows

$$
\begin{aligned}
& \phi_{\bar{p}}^{(i)}(x, y)=w+H_{\left(u_{i}, g_{i}, h_{i}\right)}(\langle 0, x, y ; \bar{p}\rangle)-x \quad \text { for } \quad(x, y) \in T, \\
& \psi_{\bar{p}}^{(i)}(x, y)=w+H_{\left(u_{i}, g_{i}, h_{i}\right)}(\langle 0, x, y ; \bar{p}\rangle)-y \quad \text { for } \quad(x, y) \in T,
\end{aligned}
$$

respectively. Then, in view of (53), for every $\bar{p} \in \mathcal{P}_{3}$, we have $\phi_{\bar{p}}:=\phi_{\bar{p}}^{(1)}=\phi_{\bar{p}}^{(2)}$ and $\psi_{\bar{p}}:=\psi_{\bar{p}}^{(1)}=\psi_{\bar{p}}^{(2)}$. Furthermore, for every $\bar{p} \in \mathcal{P}_{3}$, define $F_{\bar{p}}: T \rightarrow \mathbb{R}^{2}$ by (43).

First we show that (56) holds for some $\alpha, \beta \in(0, \infty)$. To this end, fix $x_{0} \in \mathbb{R} \backslash\{0\}$. If $x_{0} \geq w$, then in view of $(40)$, there exists $\bar{p}=\left(p_{1}, p_{2}, p_{3}\right) \in \mathcal{P}_{3}$ such that $x_{0} \in \phi_{\bar{p}}(T)$. Moreover, applying Lemma 2.7, we obtain that the set $\left\{y \in \mathbb{R}:\left(x_{0}, y\right) \in F_{\bar{p}}(T)\right\}$ is nonempty and open. Thus, $\left(x_{0}, y_{0}\right) \in F_{\bar{p}}(T)$ for some $y_{0} \in \mathbb{R} \backslash\{0\}$. In the case where $y_{0}>0$, there is $r_{x_{0}}>0$ such that $B\left(\left(x_{0}, y_{0}\right), r_{x_{0}}\right):=\left(x_{0}-r_{x_{0}}, x_{0}+r_{x_{0}}\right) \times\left(y_{0}-r_{x_{0}}, y_{0}+r_{x_{0}}\right) \subset F_{\bar{p}}(T) \cap(0, \infty)^{2}$. Therefore, taking $(x, y) \in B\left(\left(x_{0}, y_{0}\right), r_{x_{0}}\right)$ and putting $(s, t):=F_{\bar{p}}^{-1}(x, y)$, in view of $(43)$, we get $\psi_{\bar{p}}(s, t)>0$. Hence, according to Lemma 2.1(i), for $i \in\{1,2\}$, we have

$$
\left(g_{i}\left(p_{1}+p_{2}\right)-g_{i}\left(p_{1}\right)\right) u_{i}(t-s)+g_{i}\left(p_{1}\right) u_{i}(t)<u_{i}(w)
$$

and so, applying Corollary 2.2(i), we obtain

$$
\begin{aligned}
& u_{i}\left(\phi_{\bar{p}}(s, t)+s\right) \\
& \quad=\frac{1}{g_{i}\left(p_{1}\right)}\left[u_{i}(w)-\left(1-g_{i}\left(p_{1}+p_{2}\right)\right) u_{i}\left(\psi_{\bar{p}}(s, t)\right)-\left(g_{i}\left(p_{1}+p_{2}\right)-g_{i}\left(p_{1}\right)\right) u_{i}\left(\phi_{\bar{p}}(s, t)\right)\right] \\
& \quad=\frac{1}{g_{i}\left(p_{1}\right)}\left[u_{i}(w)-\left(1-g_{i}\left(p_{1}+p_{2}\right)\right) u_{i}(y)-\left(g_{i}\left(p_{1}+p_{2}\right)-g_{i}\left(p_{1}\right)\right) u_{i}(x)\right] .
\end{aligned}
$$

Thus, setting $a:=\frac{g_{1}\left(p_{1}\right)-g_{1}\left(p_{1}+p_{2}\right)}{g_{1}\left(p_{1}\right)}, A:=\frac{g_{2}\left(p_{1}\right)-g_{2}\left(p_{1}+p_{2}\right)}{g_{2}\left(p_{1}\right)}, b:=\frac{g_{1}\left(p_{1}+p_{2}\right)-1}{g_{1}\left(p_{1}\right)}$, $B:=\frac{g_{2}\left(p_{1}+p_{2}\right)-1}{g_{2}\left(p_{1}\right)}, c:=\frac{u_{1}(w)}{g_{1}\left(p_{1}\right)}$ and $C:=\frac{u_{2}(w)}{g_{2}\left(p_{1}\right)}$, for every $(x, y) \in B\left(\left(x_{0}, y_{0}\right), r_{x_{0}}\right)$, we get

$$
u_{1}^{-1}\left(a u_{1}(x)+b u_{1}(y)+c\right)=u_{2}^{-1}\left(A u_{2}(x)+B u_{2}(y)+C\right) .
$$

Therefore, a function

satisfies Eq. (46) with

$$
f:=u_{2} \circ u_{1}^{-1}
$$

$$
D:=\left\{\left(u_{1}(x), u_{1}(y)\right):(x, y) \in B\left(\left(x_{0}, y_{0}\right), r_{x_{0}}\right)\right\} .
$$

Moreover, as $u_{1}$ is a continuous injection, $D$ is open and connected. Thus, according to Lemma 2.8 , there exist $\alpha_{x_{0}} \in \mathbb{R} \backslash\{0\}$ and $\beta_{x_{0}} \in \mathbb{R}$ such that

$$
f(x)=\alpha_{x_{0}} x+\beta_{x_{0}} \text { for } \quad x \in u_{1}\left(\left(x_{0}-r_{x_{0}}, x_{0}+r_{x_{0}}\right)\right) .
$$


Hence, in view of (57), we get

$$
u_{2}(x)=\alpha_{x_{0}} u_{1}(x)+\beta_{x_{0}} \text { for } \quad x \in\left(x_{0}-r_{x_{0}}, x_{0}+r_{x_{0}}\right) .
$$

In the case where $y_{0}<0$ there exists $r_{x_{0}}>0$ such that

$$
B\left(\left(x_{0}, y_{0}\right), r_{x_{0}}\right) \subset F_{\bar{p}}(T) \cap[(0, \infty) \times(-\infty, 0)] .
$$

Furthermore, arguing as previously, we obtain that the function $f$, defined by (57), satisfies Eq. (46) with $a, A, c, C$ and $D$ as above, $b:=-\frac{h_{1}\left(p_{3}\right)}{g_{1}\left(p_{1}\right)}$ and $B:=-\frac{h_{2}\left(p_{3}\right)}{g_{2}\left(p_{1}\right)}$. Hence, (58) holds with some $\alpha_{x_{0}} \in \mathbb{R} \backslash\{0\}$ and $\beta_{x_{0}} \in \mathbb{R}$.

If $x_{0}<w$, then in view of (41), there exists $\bar{p} \in \mathcal{P}_{3}$ such that $x_{0} \in \psi_{\bar{p}}(T)$. Thus, repeating the previous procedure, we conclude that (58) is satisfied with some $\alpha_{x_{0}} \in \mathbb{R} \backslash\{0\}$ and $\beta_{x_{0}} \in \mathbb{R}$. .

In this way we have proved that for every $x \in \mathbb{R} \backslash\{0\}$ there exist $\alpha_{x} \in \mathbb{R} \backslash\{0\}$, $\beta_{x} \in \mathbb{R}$ and $r_{x} \in(0, \infty)$ such that $(50)$ is valid. Thus, as $u_{1}$ and $u_{2}$ are strictly increasing and continuous, with $u_{1}(0)=u_{2}(0)=0$, applying Lemma 2.9 with $I=(0, \infty)$ and then with $I=(-\infty, 0)$, we obtain that there exist $\alpha, \beta \in(0, \infty)$ such that (56) holds.

In the remaining part of the proof we consider two cases:

1. $w=0$,

2. $w>0$.

Case 1 Let $\bar{p}=\left(p_{1}, p_{2}, p_{3}\right) \in \mathcal{P}_{3}$ and $y \in(0, \infty)$. Then, according to Lemma $2.5, \phi_{\bar{p}}(x, y)=0$ for some $x \in(0, y)$. Thus, in view of (16), we have $\psi_{\bar{p}}(x, y)=x-y<0$. So, applying Lemma 2.1(ii) and Corollary 2.2(ii), we obtain

$$
h_{i}\left(p_{3}\right) u_{i}(x-y)+g_{i}\left(p_{1}\right) u_{i}(x)=0 \text { for } i \in\{1,2\} .
$$

Hence, considering (56), we get

$$
\frac{h_{2}\left(p_{3}\right)}{g_{2}\left(p_{1}\right)}=-\frac{u_{2}(x)}{u_{2}(x-y)}=-\frac{\beta}{\alpha} \frac{u_{1}(x)}{u_{1}(x-y)}=\frac{\beta}{\alpha} \frac{h_{1}\left(p_{3}\right)}{g_{1}\left(p_{1}\right)} .
$$

Since $\bar{p}=\left(p_{1}, p_{2}, p_{3}\right) \in \mathcal{P}_{3}$ is fixed arbitrarily, this means that

$$
\frac{h_{2}\left(p_{3}\right)}{h_{1}\left(p_{3}\right)}=\frac{\beta}{\alpha} \frac{g_{2}\left(p_{1}\right)}{g_{1}\left(p_{1}\right)} \quad \text { for } \quad p_{1}, p_{3} \in(0,1), p_{1}+p_{3}<1 \text {. }
$$

Thus, taking $q_{1}, q_{2} \in(0,1)$ and $p \in\left(0, \min \left\{1-q_{i}: i \in\{1,2\}\right\}\right)$, we obtain

$$
\frac{h_{2}\left(q_{1}\right)}{h_{1}\left(q_{1}\right)}=\frac{\beta}{\alpha} \frac{g_{2}(p)}{g_{1}(p)}=\frac{h_{2}\left(q_{2}\right)}{h_{1}\left(q_{2}\right)},
$$

which means that the function $h_{1} / h_{2}$ is constant on $(0,1)$. Since, for $i \in\{1,2\}$, $h_{i}$ is continuous, with $h_{i}(1)=1$, this implies that $h_{1}=h_{2}$. Furthermore, from (59) we derive that $\frac{g_{2}(p)}{g_{1}(p)}=\frac{\alpha}{\beta}$ for $p \in(0,1)$. Therefore, using the fact that, for $i \in\{1,2\}, g_{i}$ is continuous and $g_{i}(1)=1$, we get $\beta=\alpha$. Hence $g_{1}=g_{2}$ and, considering (56), we obtain (54). This completes the proof in the case $w=0$. 
Case 2 Fix $\bar{p}=\left(p_{1}, p_{2}, p_{3}\right) \in \mathcal{P}_{3}$ and $y \in(0, w)$. Then, in view of Lemma 2.5, there exists $x \in(0, y)$ such that $\phi_{\bar{p}}(x, y)=w$. Moreover, making use of (16), we get $\psi_{\bar{p}}(x, y)=w+x-y>0$. Thus, applying Lemma 2.1(i) and Corollary 2.2(i), for $i \in\{1,2\}$, we obtain

$\left(1-g_{i}\left(p_{1}+p_{2}\right)\right) u_{i}(w+x-y)+\left(g_{i}\left(p_{1}+p_{2}\right)-g_{i}\left(p_{1}\right)\right) u_{i}(w)+g_{i}\left(p_{1}\right) u_{i}(w+x)=u_{i}(w)$.

Hence

$$
\frac{1-g_{i}\left(p_{1}+p_{2}\right)}{g_{i}\left(p_{1}\right)}=\frac{u_{i}(w)-u_{i}(w+x)}{u_{i}(w+x-y)-u_{i}(w)} \quad \text { for } \quad i \in\{1,2\} .
$$

Therefore, since $\bar{p}=\left(p_{1}, p_{2}, p_{3}\right) \in \mathcal{P}_{3}$ is fixed arbitrarily, in view of (56), we get

$$
\frac{1-g_{1}\left(p_{1}+p_{2}\right)}{g_{1}\left(p_{1}\right)}=\frac{1-g_{2}\left(p_{1}+p_{2}\right)}{g_{2}\left(p_{1}\right)} \text { for } p_{1}, p_{2} \in(0,1), p_{1}+p_{2}<1 \text {. }
$$

Thus, as $g_{1}$ and $g_{2}$ are continuous, taking $p_{1} \in(0,1)$ and letting $p_{2} \rightarrow 0^{+}$, we obtain that $g_{1}\left(p_{1}\right)=g_{2}\left(p_{1}\right)$. Since $g_{i}(0)=0$ and $g_{i}(1)=1$ for $i \in\{1,2\}$, this implies that $g_{1}=g_{2}$.

Suppose that $\bar{p}=\left(p_{1}, p_{2}, p_{3}\right) \in \mathcal{P}_{3}$ is such that (28) holds for some $(x, y) \in$ $T$. Then, in view of Lemma 2.1(ii) and Corollary 2.2(ii), for $i \in\{1,2\}$, we get $h_{i}\left(p_{3}\right) u_{i}\left(\psi_{\bar{p}}(x, y)\right)+\left(g_{i}\left(p_{1}+p_{2}\right)-g_{i}\left(p_{1}\right)\right) u_{i}\left(\phi_{\bar{p}}(x, y)\right)+g_{i}\left(p_{1}\right) u_{i}\left(\phi_{\bar{p}}(x, y)+x\right)=u_{i}(w)$. Since $g_{1}=g_{2}$, making use of (56), from the last equality one can easily derive that

$$
h_{2}\left(p_{3}\right)=\frac{\beta}{\alpha} h_{1}\left(p_{3}\right) .
$$

Now, if $\lim _{x \rightarrow \infty} u_{1}(x)=\infty$, then applying Lemma 2.3(a), we conclude that (60) holds for every $p_{3} \in(0,1)$. Since, for $i \in\{1,2\}, h_{i}$ is continuous with $h_{i}(1)=1$, letting in (60) $p_{3} \rightarrow 1^{-}$we obtain that $\beta=\alpha$. Thus, in view of $(56)$ and (60), we get (54) and $h_{1}=h_{2}$, respectively. If $L:=\lim _{x \rightarrow \infty} u_{1}(x)<\infty$, then according to Lemma 2.3(b), (60) holds for every $p_{3} \in(0,1)$ such that $g_{1}\left(1-p_{3}\right)>u_{1}(w) / L$. As $h_{1}$ and $h_{2}$ are continuous, this implies (55).

Theorem 3.2. Let $w \in[0, \infty)$. Assume that, for $i \in\{1,2\}, u_{i}: \mathbb{R} \rightarrow \mathbb{R}$ is a strictly increasing continuous function with $u_{i}(0)=0$ and $g_{i}, h_{i}:[0,1] \rightarrow[0,1]$ are strictly increasing continuous probability distortion functions. Furthermore, let $H_{\left(u_{i}, g_{i}, h_{i}\right)}$ for $i \in\{1,2\}$ be the functionals of equivalent utility, defined by (5). If

(i) $g_{1}=g_{2}, h_{1}=h_{2}$ and (54) holds with some $\alpha \in(0, \infty)$ or

(ii) $w>0, L:=\lim _{x \rightarrow \infty} u_{1}(x)<\infty, g_{1}=g_{2}$ and (55), (56) hold with some $\alpha, \beta \in(0, \infty)$,

then

$$
H_{\left(u_{1}, g_{1}, h_{1}\right)}(X)=H_{\left(u_{2}, g_{2}, h_{2}\right)}(X) \quad \text { for } \quad X \in \mathcal{X}_{+} .
$$


Proof. Since the generalized Choquet integral is positively homogeneous (cf. $[9$, Lemma 1]), in view of (5), in the case of (i), we get

$$
\begin{gathered}
E_{g_{2} h_{2}}\left[u_{2}\left(w+H_{\left(u_{1}, g_{1}, h_{1}\right)}(X)-X\right)\right]=\alpha E_{g_{1} h_{1}}\left[u_{1}\left(w+H_{\left(u_{1}, g_{1}, h_{1}\right)}(X)-X\right)\right] \\
=\alpha u_{1}(w)=u_{2}(w) \text { for } X \in \mathcal{X}_{+},
\end{gathered}
$$

which implies (61).

Assume that (ii) holds and fix $X \in \mathcal{X}_{+}$. We claim that

$$
P\left(X>w+H_{\left(u_{1}, g_{1}, h_{1}\right)}(X)\right) \leq 1-g_{1}^{-1}\left(u_{1}(w) / L\right) .
$$

Suppose that this is not true. Then, as the space $(\Omega, \Sigma, P)$ is non-atomic, there exists $B \in \Sigma$ such that

$$
B \subset A:=\left\{X>w+H_{\left(u_{1}, g_{1}, h_{1}\right)}(X)\right\}
$$

and

$$
1-g_{1}^{-1}\left(u_{1}(w) / L\right)<P(B)<P(A) .
$$

Hence, setting

$$
\bar{X}:=w \cdot \mathbb{1}_{A \backslash B}+\left(w+H_{\left(u_{1}, g_{1}, h_{1}\right)}(X)\right) \cdot \mathbb{1}_{B},
$$

we get $\bar{X} \leq X$ which, in view of (9), gives

$$
H_{\left(u_{1}, g_{1}, h_{1}\right)}(\bar{X}) \leq H_{\left(u_{1}, g_{1}, h_{1}\right)}(X) .
$$

Furthermore, we have

$$
\bar{X}=\left\langle 0, w, w+H_{\left(u_{1}, g_{1}, h_{1}\right)}(X) ; \bar{p}\right\rangle \in \mathcal{X}_{0}^{(3)},
$$

where

$$
\bar{p}:=(1-P(A), P(A \backslash B), P(B)) .
$$

Let $T$ be of the form $(13)$ and let $\phi_{\bar{p}}^{(1)}, \psi_{\bar{p}}^{(1)}: T \rightarrow \mathbb{R}$ be given by

$$
\begin{aligned}
& \phi_{\bar{p}}^{(1)}(x, y)=w+H_{\left(u_{1}, g_{1}, h_{1}\right)}(\langle 0, x, y ; \bar{p}\rangle)-x \quad \text { for } \quad(x, y) \in T, \\
& \psi_{\bar{p}}^{(1)}(x, y)=w+H_{\left(u_{1}, g_{1}, h_{1}\right)}(\langle 0, x, y ; \bar{p}\rangle)-y \quad \text { for } \quad(x, y) \in T,
\end{aligned}
$$

respectively. Then, considering (8), (64) and (65), we obtain

$$
\begin{aligned}
& \psi_{\bar{p}}^{(1)}\left(w, w+H_{\left(u_{1}, g_{1}, h_{1}\right)}(X)\right) \\
& \quad=w+H_{\left(u_{1}, g_{1}, h_{1}\right)}(\bar{X})-\left(w+H_{\left(u_{1}, g_{1}, h_{1}\right)}(X)\right) \\
& \quad=H_{\left(u_{1}, g_{1}, h_{1}\right)}(\bar{X})-H_{\left(u_{1}, g_{1}, h_{1}\right)}(X) \leq 0 \leq H_{\left(u_{1}, g_{1}, h_{1}\right)}(\bar{X}) \\
& \quad=\phi_{\bar{p}}^{(1)}\left(w, w+H_{\left(u_{1}, g_{1}, h_{1}\right)}(X)\right) .
\end{aligned}
$$


Therefore, according to Lemma 2.3(c), we have $g_{1}(1-P(B))>u_{1}(w) / L$ and so

$$
P(B)<1-g_{1}^{-1}\left(u_{1}(w) / L\right),
$$

which contradicts (63). In this way $(62)$ is proved.

It follows from (62) that

$$
P\left(-u_{2}\left(w+H_{\left(u_{1}, g_{1}, h_{1}\right)}(X)-X\right)>t\right) \leq 1-g_{1}^{-1}\left(u_{1}(w) / L\right) \text { for } t \in[0, \infty) .
$$

Thus, in view of (4), (55) and (56), we get

$$
\begin{aligned}
& E_{h_{2}}\left[\max \left\{-u_{2}\left(w+H_{\left(u_{1}, g_{1}, h_{1}\right)}(X)-X\right), 0\right\}\right] \\
& \quad=\int_{0}^{\infty} h_{2}\left(P\left(-u_{2}\left(w+H_{\left(u_{1}, g_{1}, h_{1}\right)}(X)-X\right)>t\right)\right) d t \\
& \quad=\frac{\beta}{\alpha} \int_{0}^{\infty} h_{1}\left(P\left(-\alpha u_{1}\left(w+H_{\left(u_{1}, g_{1}, h_{1}\right)}(X)-X\right)>t\right)\right) d t \\
& \quad=\beta \int_{0}^{\infty} h_{1}\left(P\left(-u_{1}\left(w+H_{\left(u_{1}, g_{1}, h_{1}\right)}(X)-X\right)>t\right)\right) d t \\
& =\beta E_{h_{1}}\left[\max \left\{-u_{1}\left(w+H_{\left(u_{1}, g_{1}, h_{1}\right)}(X)-X\right), 0\right\}\right] .
\end{aligned}
$$

Furthermore, since the Choquet integral is positively homogeneous, making use of (56), we obtain

$$
\begin{aligned}
& E_{g_{2}}\left[\max \left\{u_{2}\left(w+H_{\left(u_{1}, g_{1}, h_{1}\right)}(X)-X\right), 0\right\}\right] \\
= & \beta E_{g_{2}}\left[\max \left\{u_{1}\left(w+H_{\left(u_{1}, g_{1}, h_{1}\right)}(X)-X\right), 0\right\}\right] .
\end{aligned}
$$

Thus, as $g_{1}=g_{2}$, considering (5) and (6), we conclude that

$$
\begin{aligned}
& E_{g_{2} h_{2}}\left[u_{2}\left(w+H_{\left(u_{1}, g_{1}, h_{1}\right)}(X)-X\right)\right] \\
& \quad=\beta E_{g_{1} h_{1}}\left[u_{1}\left(w+H_{\left(u_{1}, g_{1}, h_{1}\right)}(X)-X\right)\right]=\beta u_{1}(w)=u_{2}(w)
\end{aligned}
$$

and so $H_{\left(u_{1}, g_{1}, h_{1}\right)}(X)=H_{\left(u_{2}, g_{2}, h_{2}\right)}(X)$.

From Theorems 3.1 and 3.2 we derive the following result.

Theorem 3.3. Let $w \in[0, \infty)$. Assume that, for $i \in\{1,2\}, u_{i}: \mathbb{R} \rightarrow \mathbb{R}$ is a strictly increasing continuous function with $u_{i}(0)=0$ and $g_{i}, h_{i}:[0,1] \rightarrow[0,1]$ are strictly increasing continuous probability distortion functions. Moreover, let $H_{\left(u_{i}, g_{i}, h_{i}\right)}$ for $i \in\{1,2\}$ be the functionals of equivalent utility, defined by (5). If (53) holds then

$$
H_{\left(u_{1}, g_{1}, h_{1}\right)}(X)=H_{\left(u_{2}, g_{2}, h_{2}\right)}(X) \quad \text { for } \quad X \in \mathcal{X}_{+} .
$$

Remark 3.4. According to Theorem 3.3, every functional of equivalent utility, defined by (5), can be uniquely extended from the family $\mathcal{X}_{0}^{(3)}$ to $\mathcal{X}_{+}$. It has been already mentioned, in the Introduction, that such a functional need not be uniquely extended from the family of all binary risks. We complete the paper with a suitable example. 
Example 3.5. Let $w \in[0, \infty)$ and let $u_{1}, u_{2}: \mathbb{R} \rightarrow \mathbb{R}$ be of the form

$$
u_{1}(x)=x \text { for } x \in \mathbb{R}
$$

and

$$
u_{2}(x)= \begin{cases}\sqrt{w}-\sqrt{w-x} & \text { for } x \in(-\infty, w], \\ \sqrt{w}+\sqrt{x-w} & \text { for } x \in(w, \infty),\end{cases}
$$

respectively. Furthermore, let $g_{i}, h_{i}:[0,1] \rightarrow[0,1]$ for $i \in\{1,2\}$ be given by

$$
g_{1}(p)=h_{1}(p)=p \quad \text { for } \quad p \in[0,1]
$$

and

$$
g_{2}(p)=h_{2}(p)=\frac{\sqrt{p}}{\sqrt{p}+\sqrt{1-p}} \text { for } p \in[0,1] .
$$

Then, according to [3, Theorems 3.1-3.2], $H_{\left(u_{1}, g_{1}, h_{1}\right)}$ and $H_{\left(u_{2}, g_{2}, h_{2}\right)}$ coincide on the family of all binary risks. On the other hand, since $\lim _{x \rightarrow \infty} u_{1}(x)=\infty$, applying Theorem 3.1, we conclude that $H_{\left(u_{1}, g_{1}, h_{1}\right)}$ and $H_{\left(u_{2}, g_{2}, h_{2}\right)}$ do not coincide on $\mathcal{X}_{0}^{(3)}$.

Open Access. This article is distributed under the terms of the Creative Commons Attribution 4.0 International License (http://creativecommons.org/licenses/by/4.0/), which permits unrestricted use, distribution, and reproduction in any medium, provided you give appropriate credit to the original author(s) and the source, provide a link to the Creative Commons license, and indicate if changes were made.

Publisher's Note Springer Nature remains neutral with regard to jurisdictional claims in published maps and institutional affiliations.

\section{References}

[1] Bowers, N.L., Gerber, H.U., Hickman, J.C., Jones, D.A., Nesbitt, C.J.: Actuarial Mathematics. The Society of Actuaries, Itasca (1986)

[2] Bühlmann, H.: Mathematical Models in Risk Theory. Springer, New York (1970)

[3] Chudziak, J.: On functional equations stemming from actuarial mathematics. Aequ. Math. 92, 471-486 (2018)

[4] Chudziak, J.: On existence and uniqueness of the principle of equivalent utility under Cumulative Prospect Theory. Insur. Math. Econ. 79, 243-246 (2018)

[5] Chudziak, J.: On applications of inequalities for quasideviation means in actuarial mathematics. Math. Inequal. Appl. 21, 601-610 (2018)

[6] Chudziak, M., Sobek, B.: Generalized Pexider equation on an open domain. Results Math. 71, 1359-1372 (2017)

[7] Heilpern, S.: A rank-dependent generalization of zero utility principle. Insur. Math. Econ. 33, 67-73 (2003)

[8] Kaas, R., Goovaerts, M., Dhaene, J., Denuit, M.: Modern Actuarial Risk Theory. Springer, Berlin (2008)

[9] Kałuszka, M., Krzeszowiec, M.: Pricing insurance contracts under Cumulative Prospect Theory. Insur. Math. Econ. 50, 159-166 (2012)

[10] Kałuszka, M., Krzeszowiec, M.: On iterative premium calculation principles under Cumulative Prospect Theory. Insur. Math. Econ. 52, 435-440 (2013) 
[11] Kuczma, M.: An Introduction to the Theory of Functional Equations and Inequalities. Cauchy's Equation and Jensen's Inequality, 2nd edn. Birkhäuser, Berlin (2009)

[12] Rachev, S.T., Klebanov, L.B., Stoyanov, S.V., Fabozzi, F.J.: The Methods of Distances in the Theory of Probability and Statistics. Springer, New York (2013)

[13] Radó, F., Baker, J.A.: Pexider's equation and aggregation of allocations. Aequ. Math. 32, 227-239 (1987)

[14] Rolski, T., Schmidli, H., Schmidt, V., Teugels, J.: Stochastic Processes for Insurance and Finance. Wiley, New York (1999)

Jacek Chudziak

Faculty of Mathematics and Natural Sciences

University of Rzeszów

ul. Pigonia 1

35-310 Rzeszow

Poland

e-mail: chudziak@ur.edu.pl

Received: March 30, 2018 